\title{
QoS provisioning in LTE-A networks with relay nodes
}

\author{
G. Piro, Student Member, IEEE, L.A. Grieco, Senior Member, IEEE, G. Boggia, Senior Member, IEEE, \\ and P. Camarda
}

\begin{abstract}
In the vision of heterogeneous networks, the Quality of Service provided by classic cellular systems is improved thanks to a jointly adoption of macro and small-range cells (i.e., micro, pico, and femtocells) working in the same area. This rationale has been also assimilated by 3GPP, which now proposes its possible exploitation in the upcoming Long Term Evolution-Advanced architecture. In this context, the wireless relay technology represents an interesting solution for extending the coverage of a macrocell by means of a relay node which offers wireless connectivity to mobile users and, at the same time, is connected to the eNode-B (and hence to the backbone) through a wireless backhaul link. But, the standard does not specify any optimal resource allocation strategy for the two hop path formed by the user and the backhaul links, which is indeed very relevant to satisfy the level of QoS expected by end users. To this aim, in the present contribution, we present a novel scheduling strategy, conceived as an extension of the recently proposed TwoLevel Scheduler approach, able to effectively support real-time applications in the presence of relay nodes. Its performance has been tested in several network scenarios that involve a variable number of users hosting real-time multimedia applications and best effort flows. A comparison with respect to Proportional Fair and Logarithmic strategies has been also provided, showing that the proposed approach is able to greatly reduce the Packet Loss Ratio experienced by multimedia applications, at the expense of a slight impairment of the throughput of the best effort flows.
\end{abstract}

Index Terms-LTE-A, relay nodes, Frame Level Scheduler, QoS, real-time services

\section{INTRODUCTION}

The Third Generation Partnership Project (3GPP) has conceived, within the emerging LTE Advanced (LTE-A) architecture, several innovative technological solutions, such as carrier aggregation, enhanced multi-antenna support, coordinated multi-point transmission techniques, relaying, and multi-user multiple input multiple output communications, thus allowing next generation cellular systems to work very close to the Shannon limit [1].

In addition, the idea to deploy a heterogeneous network composed by macro and small-range (i.e., micro, pico, and femto) cells as been also introduced. Micro and pico cells could be exploited for enhancing coverage and capacity in some regions inside the macro one [1]. Whereas, femtocells have been devised for offering broadband services in indoor (home and offices) and outdoor scenarios with a very limited geographical coverage [2].

Besides aforementioned solutions, the adoption of the relay technology could be very useful for extending and enforcing

Authors are with the "DEE - Dip. di Elettrotecnica ed Elettronica", Politecnico di Bari, v. Orabona, 4 - 70125, Bari, Italy. e-mail: \{g.piro,a.grieco,g.boggia,camarda\}@poliba.it. the coverage of the base station of a macrocell, namely the evolved Node B (eNB), especially in remote areas where mobile terminals experience bad channel conditions. To this aim, the novel relay node is introduced in the cellular architecture to bind mobile terminals, along the so called user link, while maintaining a connection to the eNB through an ad-hoc backhaul link. Note that in LTE-A specifications, when in the presence of a relay node, the eNB of the macrocell is also referred to as Donor eNB (DeNB). The Multicast Broadcast Single-Frequency Network (MBSFN) frame structure, which has been initially conceived for offering enhanced multimedia services (such as mobile TV broadcasting) over the LTE infrastructure, can be exploited to handle simultaneously communications along user and backhaul links [1]. In particular, the communication among relay nodes and DeNBs is allowed only during specific time slots (i.e., MBSFN time slots). The remaining time slots (i.e., not-MBSFN time slots) can be used by relay nodes and DeNBs for exchanging packets with mobile terminals.

The standard does not specify any resource allocation strategy for the two-hop path formed by the user and the backhaul links, thus leaving vendors and operators free to implement own custom solutions. To the best of our knowledge, at the time of this writing, only few contributions are available in literature on this subject [3]-[5]. They propose very basic schemes that are unable to offer any sharp QoS bounds to real-time applications.

To bridge this gap, the present contribution proposes a novel approach, conceived by properly tailoring the recently devised Two Level Scheduler algorithm [6][7], which is able to allocate radio resources on both user and backhaul links in order to satisfy the requirements of real-time applications.

The LTE-Sim simulator [8] has been adopted for demonstrating that, in a LTE-A scenario composed by macro cells, relay nodes, a variable number of users, and in the presence of both multimedia and best effort applications, the devised strategy is able to greatly outperform Proportional Fair (PF) and Logarithmic (LOG) rule algorithms [9] in terms of Packet Loss Ratio (PLR) of video and VoIP real-time flows, at the expense of a a slight reduction of the throughput of best effort services.

\section{THE PROPOSED SCHEDULING STRATEGY}

\section{A. The Two-Level Scheduler}

The resource allocation strategy we present herein has been designed by properly tailoring the Two Level Scheduler algorithm presented in [6], with the aim of guaranteeing 
bounded delays to multimedia flows also in the presence of two-hop wireless relaying paths.

The Two-Level Scheduler has been originally devised for guaranteeing sharp delay bounds to real-time services in the LTE downlink. It is built on two distinct levels. At the highest one, a resource allocation strategy, namely Frame Level Scheduler (FLS), defines on the long run (i.e., in a single frame lasting $T_{f}$ ) the amount of data that should be transmitted by each data source to satisfy its delay constraint. The lowest level, instead, work on a time-slot basis to allocate radio resources by taking into account the channel status as well as the constraints imposed by FLS.

Packets generated by a given application and directed to a mobile terminal are enqueued, at the base station side, into a specific queue. Let $q_{i}(k), u_{i}(k)$, and $d_{i}(k)$ be the amount of data in the queue associated to the $i$-th flow at the beginning of the $k$-th frame, the amount of data to be transmitted during the $k$-th frame, and the amount of data generated by the $i$ th flow during the $k$-th frame, respectively. In FLS, $u_{i}(k)$ is computed with the following control law ${ }^{1}$ :

$u_{i}(k)=q_{i}(k) \sum_{n=2}^{M_{i}}\left[q_{i}(k-n+1)-q_{i}(k-n+2)-u_{i}(k-n+1) c_{i}(n)\right.$

In this way, as demonstrated in [6], the upper bound of the queuing delay, $\tau_{i}$, is equal to: $\tau_{i}=\left(M_{i}+1\right) T_{f}$.

Starting from the beginning of each frame, the lower level scheduler allocates radio resources only to real-time flows, allowing them to transmit the quota of data $u_{i}(k)$ defined by FLS. As soon as a real time source has transmitted its amount of data, $u_{i}(k)$, it looses the opportunity to transmit until the beginning of next frame. To achieve a high level of fairness among multimedia flows, the lower layer scheduler uses the $\mathrm{PF}$ algorithm [9]. Then, radio resources left free by real-time flows will be assigned to best effort applications even using the PF scheduler, thus providing a high degree of fairness also to these kind of flows.

\section{B. Adapting the Two-level Scheduler to the relay technology}

The aforementioned approach can be straightly cast in a two-hop relay path by splitting in two parts the maximum delay on the access network, $\tau_{i}$ (i.e., from the DeNB to the terminal), as follows:

$$
\tau_{i}=\tau_{i, \text { DeNB->relay }}+\tau_{i, \text { relay }->U E} .
$$

where $\tau_{i, D e N B->\text { relay }}$ and $\tau_{i \text {,relay }->U E}$ are the delay on the backhaul and on the user links, respectively, as shown in Fig. 1.

In line with this premise, we conceived a distributed version of the Two-Level Scheduler in which every DeNB and relay node execute autonomously the resource allocation strategy presented in Sec. II-A, by imposing its own target delay (i.e., $\tau_{i, \text { DeNB }}->$ relay and $\left.\tau_{i, \text { relay }->U E}\right)$.

It is worth to remark that, in the FLS rationale, the amount of data that each station can receive within a frame does not depend on the structure of the frame itself; so that, this algorithm can be easily cast in any MBSFN configuration.

${ }^{1}$ Details about the design of the closed control loop, scalability issues, system stability, and setting of $c_{i}$ coefficients are in [6].

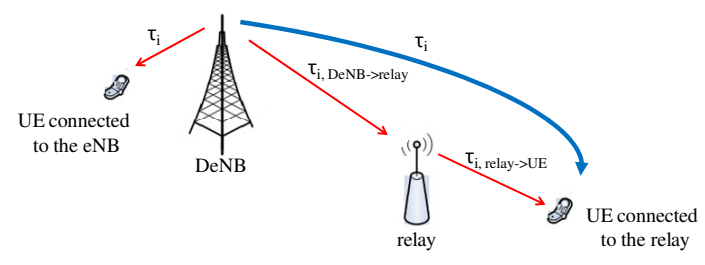

Fig. 1. Target delays in a multi-hop connection.

\section{Performance eVAluation}

The LTE-Sim framework [8] has been used for evaluating the performance of the proposed approach as well as for comparing it with respect to the well-known schemes PF and LOG rule [9]. In particular, we considered a realistic multicell scenario composed by 19 macrocells with radius equal to 0.5 $\mathrm{km}$. In each macrocell, there are: a variable number of users in the interval $[10,20]$, one eNB, and 6 relay nodes, placed at a distance from the eNB equal to the $80 \%$ of the cell radius.

A static frequency reuse strategy has been adopted for distributing frequencies among base stations and for guaranteeing to each of them a downlink bandwidth equal to $10 \mathrm{MHz}$. At the physical layer, we set the power transmission of the eNB and the relay node to $46 \mathrm{dBm}$ and $30 \mathrm{dBm}$, respectively. Moreover, considering $d$ the distance among nodes in kilometers, the urban channel model has been considered, where the pathloss between eNB and user or relay node is calculated as $P_{L}=128.1+37.6 \log d$ and the one between relay node and user is given by $P_{L}=103.8+20.9 \log d$.

According to [1], we set up a specific MBSFN frame structure, which is repeated without any variations every frame, where time slots number 1, 6, 7, and 10 have been classified as not-MBSFN.

As in [6], we imposed that each user receives at the same time one video flow encoded with H.264 at the average rate of $128 \mathrm{kbps}$, one VoIP flow, and one best effort flow modeled with an infinite buffer source. In our simulations we consider two different target delays, $D$, for multimedia applications equal to $60 \mathrm{~ms}$ and $100 \mathrm{~ms}$. The same value has been adopted for both video and voice flows in order to ensure that they are synchronously played out at the same user, which is of major importance in video-conferencing.

Regarding the maximum delays, we imposed that:

$$
\tau_{i}^{D R}=\tau_{i}^{R U}=\tau_{i} / 2 .
$$

Furthermore, $M_{i}$ and $c_{i}$ coefficients have been set as in [6]. Finally, PF and LOG rule algorithms have been implemented independently on both the backhaul and user links, using the parameter settings presented in [10].

The comparison among scheduling strategies has been devised on the basis of several performance indexes such as the PLR of multimedia applications as well as the goodput and the fairness of best effort flows. From Fig. 2, showing the PLR of both video and VoIP flows, it is possible to observe that the PLR increases with the number of users, due to the higher network load. As expected, a lower value of the target delay implies a higher value of PLR due to a larger quota of packets violating the deadline (note that packets are deleted from the 
transmission queue when they are not transmitted within the deadline). The most important result we obtained is that the proposed approach is able to achieve the smallest PLR. This is a clear demonstration that it can greatly improve the quality of multimedia services also in the presence of relay nodes. It is worth to note that VoIP flows experience significantly smaller PLRs than the ones experienced by video flows. The reason is these kind of flows, having a lower source bit rate, get the highest priority from the PF scheduler.

To study the behavior of best-effort flows, Fig. 3 shows the aggregate goodput, defined as the rate of useful bits they successfully transmit during the whole simulation. We note that the goodput decreases as the number of users increase.

Both LOG rule and PF allocation schemes obtain a higher goodput for best-effort flows with respect to the FLS algorithm. This result was expected because, as seen in Fig. 2, LOG rule and PF provide a worse service to multimedia flows (with respect to our proposal), thus leaving a higher quota of bandwidth for best-effort flows. To gain a further insight, we evaluated the Jain Fairness Index [11], finding it is higher than 0.8 in all considered conditions. This means that all considered strategies are able to provide high levels of fairness to besteffort applications.

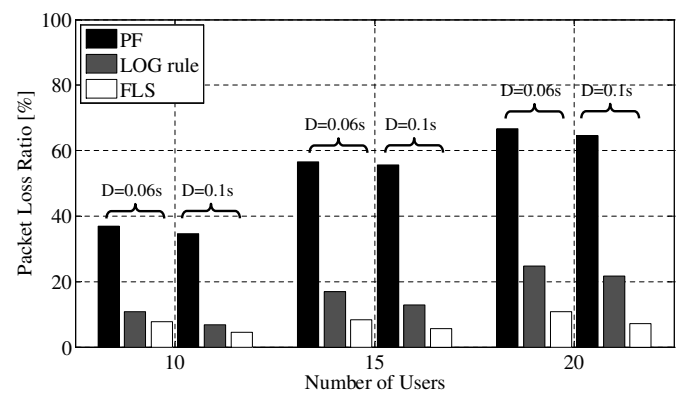

(a)

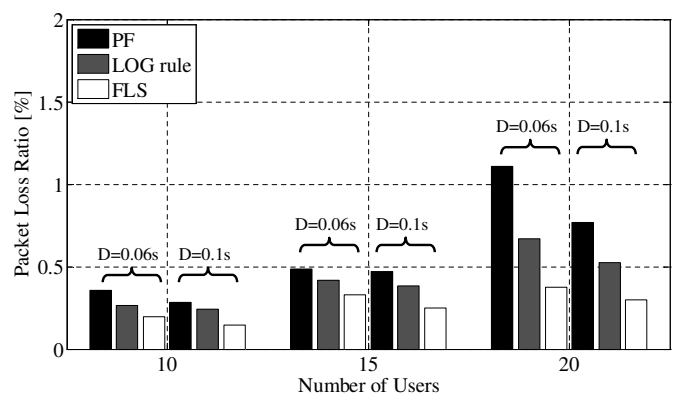

(b)

Fig. 2. PLR of (a) video and (b) VoIP flows, with target delay $D$.

\section{CONCLUSION AND FUTURE WORKS}

In this paper, we presented a novel scheduling strategy able to offer bounded delay to real-time applications in Heterogeneous Networks (HetNets) composed by macrocell and relay nodes. Its performance have been tested using LTESim, considering several network conditions and real-time multimedia applications. A comparison with respect to both PF and LOG rule strategies has been also provided. Results clearly

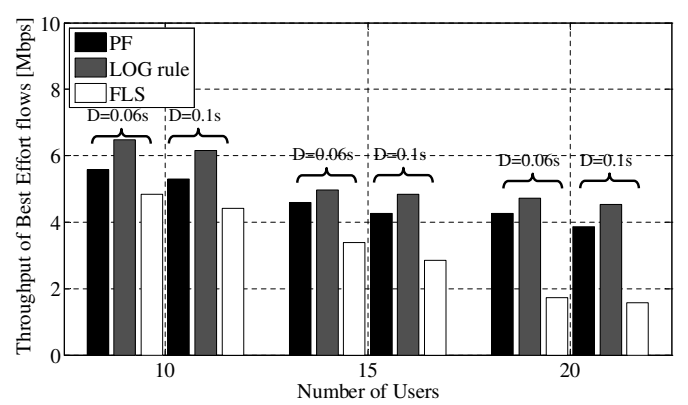

Fig. 3. Throughput of best effort flows.

show that the proposed approach is able to greatly outperform other schemes in terms of packet loss ratio achieved by video and voice over IP (VoIP) flows. In the future, we plan to test the algorithm in more complex HetNets (introducing for example also micro, pico and femtocells) and to evaluate its energy efficiency.

\section{ACKNOWLEDGMENTS}

This work was partially funded by the Apulia Region Project PS 025 (ICT supporting logistic services: a model of organized market) and the National Operative Program ERMES (Enhance Risk Management through Extended Sensors).

\section{REFERENCES}

[1] E. Dahlman, S. Parkvall, and J. Skald, $4 G$ LTE/LTE-Advanced for Mobile Broadband. Academic Press, 2011.

[2] F. Capozzi, G. Piro, L. A. Grieco, G. Boggia, and P. Camarda, "On accurate simulations of LTE femtocells using an open source simulator," EURASIP Journal on Wireless Communications and Networking, 2012, to be published.

[3] Z. Ma, W. Xiang, H. Long, and W. Wang, "Proportional Fair Resource Partition for LTE-Advanced Networks with Type I Relay Nodes," in Proc. of IEEE Int. Conf. on Commun. (ICC), Kyoto, Japan, Jun. 2011, pp. $1-5$.

[4] G. Liebl, T. de Moraes, A. Soysal, and E. Seidel, "Fair resource allocation for inband relaying in LTE-Advanced," in Proc. of Int. Workshop on Multi-Carrier Systems Solutions (MC-SS), Herrsching, Germany, May 2011, pp. $1-5$.

[5] M. Salem, A. Adinoyi, M. Rahman, H. Yanikomeroglu, D. Falconer, K. Young-Doo, K. Eungsun, and C. Yoon-Chae, "An Overview of Radio Resource Management in Relay-Enhanced OFDMA-Based Networks," IEEE Commun. Surveys and Tutorials, vol. 12, no. 3, pp. 422 -438, Apr. 2010.

[6] G. Piro, L. Grieco, G. Boggia, R. Fortuna, and P. Camarda, "Twolevel Downlink Scheduling for Real-Time Multimedia Services in LTE Networks," IEEE Trans. Multimedia, vol. 13, no. 5, pp. $1052-1065$, Oct. 2011.

[7] G. Piro, L. A. Grieco, G. Boggia, and P. Camarda, "A two-level scheduling algorithm for qos support in the downlink of lte cellular networks," in Proc. of European Wireless, EW2010, Lucca, Italy, Apr. 2010.

[8] G. Piro, L. Grieco, G. Boggia, F. Capozzi, and P. Camarda, "Simulating LTE Cellular Systems: An Open-Source Framework," IEEE Trans. Veh. Technol., vol. 60, no. 2, pp. $498-513$, Feb. 2011.

[9] F. Capozzi, G. Piro, L. A. Grieco, G. Boggia, and P. Camarda, "Downlink packet scheduling in lte cellular networks: Key design issues and a survey," IEEE Commun. Surveys Tuts., 2012.

[10] B. Sadiq, R. Madan, and A. Sampath, "Downlink scheduling for multiclass traffic in lte," EURASIP J. Wirel. Commun. Netw., vol. 2009, pp. 9-9, 2009.

[11] R. Jain, The Art of Computer Systems Performance Analysis. John Wiley \& Sons, 1991. 